\title{
Caracterización agrosocioeconómica y biofísica del área de influencia del sistema de riego Aguarongo - Zañe (Loja - Ecuador)
}

\author{
Agrosocioeconomic and biophysical characterization of the area of influence of the \\ irrigation system Aguarongo - Zañe (Loja - Ecuador)
}

\author{
María Chamba-Ontaneda ${ }^{1, *}$ \\ ${ }^{1}$ Universidad Nacional de Loja. Loja, Ecuador \\ * Autor para correspondencia: merys15@gmail.com
}

\begin{abstract}
Resumen-La caracterización de los factores biofísicos y socioeconómicos es fundamental en la planificación del desarrollo sostenible de la población. En la investigación se determinaron los aspectos biofísicos y agrosocioeconómicos del área de influencia del sistema de riego Aguarongo-Zañe. Las variables analizadas fueron factores climáticos, geología regional y local, características físico-químicas del suelo, uso actual del suelo y los aspectos agrosocioeconómicos. Los resultados obtenidos indican que el área de estudio posee un clima semihúmedo y geológicamente se ubica en la formación Sacapalca. Dentro de los aspectos socioeconómicos, las principales actividades económicas son la agricultura y ganadería no tecnificada, y los factores que impiden el desarrollo de la comunidad son los bajos precios que reciben por la venta de la producción agropecuaria. El estudio de los aspectos biofísicos y socioeconómicos permite contar con fundamentos para el diseño y planificación de proyectos de desarrollo para el bienestar comunitario.
\end{abstract}

Palabras clave-Producción, Agricultura, Proyectos de desarrollo.

\begin{abstract}
The characterization of the biophysical and socioeconomic factors is fundamental in planning the sustainable development of the population. This research determined the biophysical and agrosocioeconomic aspects of the area of influence of the Aguarongo-Zañe irrigation system. The variables analyzed were climatic factors, regional and local geology, physical-chemical characteristics of the soil, current use of the soil and agrosocioeconomic aspects. The results obtained indicate that the study area has a semi-humid climate and is geologically located in the Sacapalca formation. Within the socioeconomic aspects; the main economic activities are agriculture and nontechnical livestock, and the factors that impede the development of the community are the low prices they receive for the sale of agricultural production. The study of the biophysical and socioeconomic aspects allows to have foundations for the design and planning of development projects for community well-being.
\end{abstract}

Keywords_-Production, Agriculture, Development projects.

\section{INTRODUCCIÓN}

$\mathbf{U}^{\prime}$ n estudio biofísico y agrosocioeconómico es fundamental a la hora de planificar e implementar proyectos de desarrollo para la comunidad encaminados a mejorar el progreso en los diferentes ámbitos de desarrollo agrícola y pecuario (Flores Jibaja, 2019), en donde la sostenibilidad integra diversos componentes tales como tecnología, innovación de productos, sistemas producto-servicio e innovaciones sociales (González Insua et al., 2020) orientados a la protección y uso correcto de los recursos naturales garantizando la prevalencia en el tiempo sin alterar los ambientes para futuras generaciones (Wong Martínez, 2019).

Al hablar de sostenibilidad se hace referencia al equilibrio en función de los recursos del entorno (Zúñiga, 2011), por ende, la sostenibilidad económica se logra con una economía productiva que maneje los recursos naturales renovables a una tasa que equivale a su propia tasa de recuperación en armonía con las políticas del crecimiento económico, favoreciendo el balance entre las necesidades actuales y las futuras (Barrios et al., 2007).

La sostenibilidad del medio ambiente se fundamenta en la pluralidad cultural y en el potencial de la naturaleza en diferentes contextos de la ecología, la identidad de los pueblos y la conservación; este enfoque prioriza las prácticas agrícolas de protección, preservación de la erosión y manejo integral de los suelos (mantenimiento y fertilización), conservación de las especies e invención en los sistemas agroecológicos de elevada producción (Leff et al., 2002), es decir, fomenta una minimización de los elementos de perturbación a la 
naturaleza y mantiene un equilibrio entre los productos e insumos materiales de la población a nivel local, regional y global (Gallopín, 2003).

El desarrollo sostenible guarda una estrecha relación entre el bienestar social y el ambiente; rescata la diversidad cultural y prioriza los escenarios biofísicos como un eje integrador (Leffet al., 2002). Por tanto, el desarrollo sostenible se considera como un proceso que permuta en diversas formas de producción y consumo que permite garantizar la interacción armoniosa con la naturaleza y los seres humanos. Entre las variables que permiten cuantificar el mayor o menor desarrollo se encuentran los aspectos económicos, políticos y ambientales (Mora, 2013).

El desarrollo sostenible de cada país depende de la política y gestión integral del ambiente en coordinación del sector público, privado y la población en general. Para lograr el desarrollo sostenible. los proyectos a ejecutarse en la comunidad deben comprender aspectos ambientales, sociales y económicos encaminados a cambiar la forma de producción, distribución y consumo de los recursos naturales (Flores, 2008).

La provincia de Loja se caracteriza por ser una zona eminentemente agrícola y ganadera. En la agricultura destaca el cultivo de café, maíz, fréjol, frutas y hortalizas. La superficie dedicada a los cultivos a través del tiempo ha perdido los macros y micronutrientes esenciales para la producción, tales como el nitrógeno, fósforo, potasio, calcio, magnesio y boro. Por tanto, la productividad ha disminuido notablemente lo que incide en el incremento de necesidades básicas insatisfechas. La producción agrícola y ganadera son actividades importantes dentro de la economía del área de estudio.

No obstante, la falta de información, las políticas de desarrollo y las técnicas de explotación de los recursos en los últimos años, han demostrado ser inadecuados por no considerar las características biofísicas del entorno, lo que da como resultado problemas de erosión. Considerando que la población se dedica en mayor porcentaje a la agricultura y depende directamente de los recursos naturales se enfatiza la necesidad de diagnosticar el estado actual de los recursos para orientar las actividades económicas que permitirán un manejo integral.

A través de esta investigación se pretende contribuir con un diagnóstico de los aspectos biofísicos y económicos que permitan conocer detalladamente las características específicamente de los suelos y en función de los resultados establecer planes de manejo y fertilización de los mismos como estrategia de desarrollo sostenible para mejorar su calidad de vida en relación a un manejo adecuado del entorno natural.

\section{MATERIALES Y MÉTODOS}

\section{Área de estudio}

El área de influencia del sistema de riego está formada por los barrios Simón Bolívar, El Calvario, San Antonio, La Dolorosa, Huiñacapac y San Vicente, parroquia Chuquiribamba, cantón Loja (Figura 1). Se ubica en la Zona 17 Sur, a una altitud de $2657 \mathrm{msnm}$, en las coordenadas $0683993 \mathrm{E}$, 9514036 N. Existe un total de 99 usuarios del sistema de riego en una superficie de 98,30 ha de las cuales solamente el $81,7 \%(80,35$ ha) se encuentran bajo riego y el $19,65 \%$ no tiene riego.

\section{Aspectos físicos}

\section{Factores climáticos}

Para la determinación de los factores climáticos del área de estudio se utilizó información secundaria obtenida del INAMHI de las estaciones La Argelia, Catamayo, San Lucas y El Cisne, sobre temperatura, humedad relativa y precipitación (INAMHI, 2013).

\section{Geología regional y local}

La geología regional fue determinada mediante el uso de la Hoja geológica (Instituto Nacional de Investigación Geológico Minero Metalúrgico, 2017) a una escala de 1:500000 y las coordenadas obtenidas con el GPS. Para determinar la geología local se construyeron 6 calicatas de 1,20 m de ancho por 1,50 m de largo, con una profundidad de 1,20 m. Se obtuvieron muestras in situ que fueron llevadas al Laboratorio de Petrografía de la Universidad Nacional de Loja para su posterior análisis.

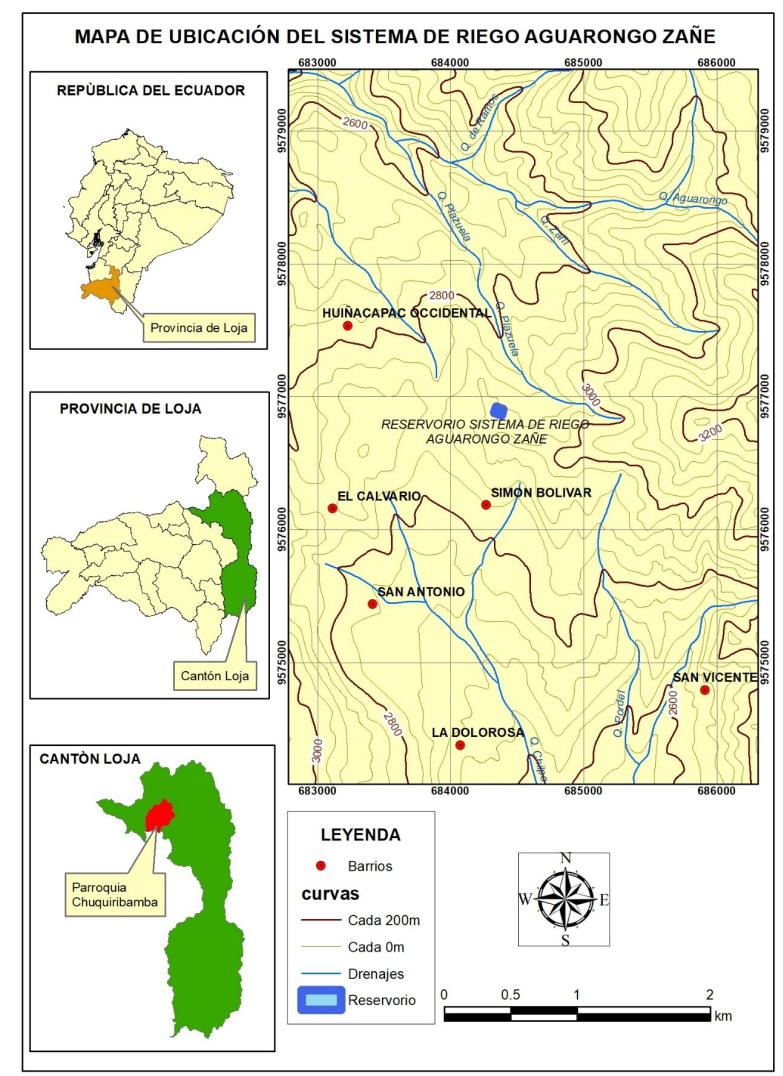

Fig. 1: Área de estudio: ubicación del Sistema de Riego Aguarongo - Zañe.

\section{Características físico-químicas del suelo}

Para determinar las características fisicoquímicas del suelo, en el mes de enero de 2020 se tomaron 6 muestras de suelo de $1 \mathrm{~kg}$ cada una, es decir, una muestra por cada sector. Los procesos que se aplicaron están en función de la Guía para la descripción de suelos de la Organización de las Naciones Unidas para la Alimentación y la Agricultura (FAO) (Vargas Rojas, 2009). 
- Características físicas del suelo: En cada muestra se evaluó el color y la textura. El color se evaluó por el método de observación con la ayuda de la tabla Munsell (Domínguez Soto et al. 2012), mientras que la textura se midió mediante el método de Bouyoucos o método del hidrómetro (Beretta et al. 2014).

- Características químicas del suelo: En las mismas muestras se evaluó el contenido de macronutrientes, el pH, la capacidad de intercambio catiónico y el contenido en materia orgánica. El contenido de los macronutrientes Nitrógeno, Fósforo y Potasio se determinó a través del método de absorción atómica (Ruales Torres, 2019), el pH por el método del potenciómetro (Ruiz Román, 2018), la capacidad de intercambio catiónico por el método de Formaldehido (Guerrero Lázaro, 2019) y el contenido de materia orgánica mediante el método de Walkley Black (Méndez Valdiviezo, 2016).

\section{Uso actual del suelo}

La información se obtuvo del Plan de Desarrollo y Ordenamiento Territorial de la Parroquia Chuquiribamba 2014 2019 y a través de la aplicación de 99 encuestas a los usuarios del Sistema de riego Aguarongo-Zañe.

\section{Aspecto socioeconómico}

El trabajo investigativo se realizó a través de etapas, que van desde la revisión de información secundaria, elaboración de los instrumentos de recolección de información de campo (encuestas, entrevistas estructuradas, etc.) y aplicación de los instrumentos para la recolección de información de campo.

1. Revisión de información secundaria. En un primer momento se revisaron documentos y datos estadísticos de diversas fuentes como: Censos de Población y Vivienda (Instituto Nacional de Estadística y Censos, 2010) para la obtención de crecimiento poblacional, distribución de la población por edades y género y población económicamente activa, así como Planes de Desarrollo y Ordenamiento Territorial de la Provincia de Loja, cantón Loja y parroquia Chuquibamba (Gobierno Autónomo Descentralizado Parroquial [GADP] Chuquiribamba y Constructora Consultora Inka Pirka, 2015), con el fin de conocer la organización y distribución de la población en el área de estudio.

2. Elaboración de instrumentos de información. Después de haber realizado las visitas respectivas a los barrios Simón Bolívar, El Calvario, San Antonio, La Dolorosa, Huiñacapac y San Vicente, se procedió a elaborar los instrumentos de recolección de información, es decir, las encuestas; los parámetros considerados en la redacción de las encuestas fueron composición familiar, actividad económica, vivienda, migración, organización social, división sexual del trabajo, salud, estructura agraria, nivel tecnológico de los procesos productivos agrícolas, pecuarios, agroindustriales y/o artesanales y factores que impiden el desarrollo agropecuario y forestal.

3. Trabajo de campo. Se aplicaron los instrumentos de información a los 99 jefes de familia usuarios del sistema de riego Aguarongo-Zañe en el mes de enero de 2020 a través de entrevista personal.

\section{RESULTADOS}

\section{Aspectos físicos}

\section{Factores climáticos}

La parroquia de Chuquiribamba posee un clima semihúmedo; tiene una precipitación que se encuentra entre $800 \mathrm{~mm}$ a $1300 \mathrm{~mm}$, en donde los cuatro primeros meses del año son los menos húmedos (Figura 2); la temperatura oscila entre $10^{\circ} \mathrm{C}$ a $20^{\circ} \mathrm{C}$ y la velocidad de viento es de $8 \mathrm{~km} / \mathrm{h}$.

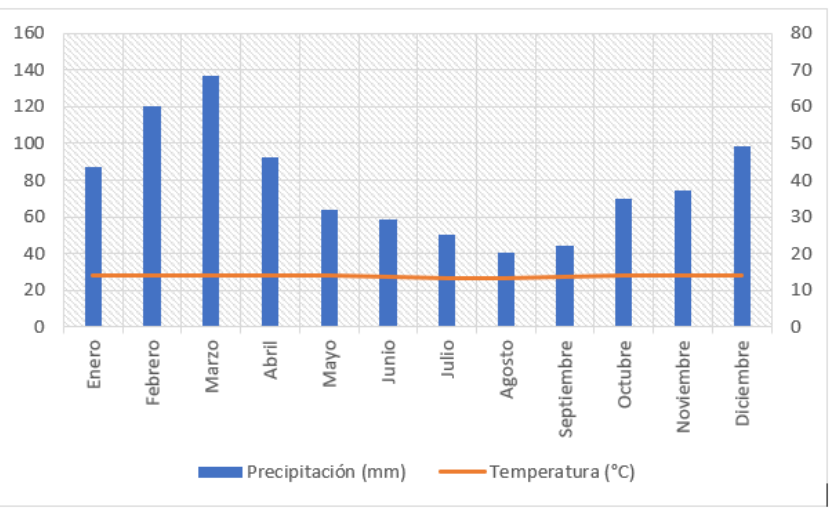

Fig. 2: Climograma del área de estudio - Sistema de Riego Aguarongo - Zañe (modificada de Gobierno Provincial de Loja Riego y Gestión Ambiental 2018).

\section{Geología regional y local}

Regionalmente, el área de estudio corresponde a la formación Sacapalca y predominan depósitos de avalanchas y "debris slope" que presentan cantos y guijarros de rocas metamórficas en matriz arenosa. Las características de la formación de acuerdo a la carta geológica indican la presencia de un depósito coluvial y en los sectores de Pichic, Zañi y Palta se identificaron bloques de material de toba andesíticas y riolíticas. Localmente, debido a la profundidad de la calicata no se encontró material rocoso de ningún tipo, y solo se pudo identificar materia orgánica.

\section{Características físico-químicas del suelo}

En función de los valores identificados en laboratorio y en función de la Pirámide Textural, al horizonte Ap le corresponde la clase textural Franco Arenoso. El valor del pH indica que los suelos son ligeramente ácidos (Tabla 1). Mediante el Carbono Orgánico se pudo determinar el porcentaje de materia orgánica presente en el suelo, en donde se pudo estimar que en el Perfil Ap se encuentra un bajo contenido de materia orgánica.

\section{Uso actual del suelo}

El área de estudio presenta $2,38 \%$ de uso agrícola, $31,68 \%$ pecuario, $1,60 \%$ agropecuario, 59,84\% conservación (bosque nativo, chaparral, luzara, bosque primario, pajonal, eucaliptos, bosque seco, ciprés y pino), 4,17\% protección, $0,12 \%$ antrópico y $0,21 \%$ improductivo. Se 
observaron tres tipos de vegetación: pastos tales como pasto azul (Poa pratensis) y pastos verdes (Brachiaria prizanta), cultivos de hortalizas, legumbres y árboles frutales y bosque.

Tabla 1: Características físicas y químicas del suelo en el área de influencia del sistema de riego Aguarongo. $\mathrm{CO}=$ carbono orgánico; $\mathrm{MO}=$ materia orgánica; $\mathrm{Al}=$ aluminio

\begin{tabular}{cc}
\hline Parámetros & Horizonte Ap \\
\hline $\mathrm{pH}$ & 5,81 \\
$\mathrm{CO} \%$ & 2,67 \\
$\mathrm{MO} \%$ & 4,62 \\
$\mathrm{Al}$ meq/100 ml suelo & 1,18 \\
$\mathrm{Al}+\mathrm{H}+$ meq/100 ml suelo & 1,34 \\
$\mathrm{P} \mathrm{ppm}$ & 36,5 \\
$\mathrm{~K}$ meq/100 g suelo & 1,37 \\
$\mathrm{~N} \%$ & 0,23 \\
\hline
\end{tabular}

\section{Aspecto socioeconómico}

La principal actividad económica que se realiza en la parroquia Chuquiribamba es la ganadería y agricultura como medio de ingresos económicos, la tasa de analfabetismo se incrementa al $10,81 \%$, por tanto, la población opta por realizar actividades pecuarias y labranza de la tierra desde muy temprana edad. En cuanto a la vivienda el $100 \%$ de los encuestados tiene una vivienda propia, en la mayoría (54\%) de los casos de tierra, mientras que el resto son de ladrillo (31\%) y tapia $(15 \%)$.

En la tenencia de tierra se debe indicar que las superficies cultivables fueron adquiridas a través de la compra y por medio de herencias familiares y se caracterizan por su superficie menor a una hectárea.

Las actividades productivas en su mayoría (70\%) son realizadas por el hombre, considerando que mayor fuerza física la presenta el hombre, mientras que el $30 \%$ de las actividades y la participación comunitaria, económica, social, administrativa y de capacitación son realizadas por la mujer.

La agricultura que se practica en el sector es tradicional, la labranza del suelo se realiza con implementos manuales tales como lampa, azadón, pico, machete, hoz, barreta, etc. La tecnología agrícola que se utiliza en el sector hace referencia al sistema de riego: el $90 \%$ utiliza el método de riego por aspersión y el $10 \%$ por goteo. La dotación del recurso hídrico para riego se realiza a través de la Junta de regantes del Sistema de Riego Aguarongo - Zañe. La fertilización del suelo se realiza en un $95 \%$ a través de abono orgánico y el $5 \%$ emplea otros tipos de fertilizantes. La preparación del suelo se realiza en un $90 \%$ con tractor y el $10 \%$ lo realiza con yunta de bueyes. Las principales plagas presentes en los cultivos son los gusanos cogolleros (Spodoptera frugiperda) y babosas (Deroceras reticulatum).

En los procesos productivos pecuarios, el $65 \%$ de los encuestados se dedican a la crianza de ganado bovino, el $15 \%$ tiene ganado porcino, el $10 \%$ ganado ovino y el $10 \%$ restante se dedica al ganado equino. Así también, la producción de animales menores la realizan el $80 \%$ de los encuestados y está dividida en $60 \%$ cuyes y el $40 \%$ de aves de corral.

En el manejo del ganado bovino, el $70 \%$ utiliza el sogueo, $20 \%$ libre y el $10 \%$ utiliza otros métodos. En cuanto a la ali- mentación, el $75 \%$ del ganado bovino se alimenta de pasto azul (Poa pratensis), el $15 \%$ con raigrás (Lolium perenne) y el $10 \%$ con kikuyo (Pennisetum clandestinum). En cuanto a la reproducción del ganado bovino, el $80 \%$ lo realiza sin mejoramiento y el $20 \%$ es mejorada, es decir con el uso de pajuelas de ganado de mayor producción de leche o carne a través de la inseminación artificial. La sanidad animal que se realiza en el sector consiste en la aplicación de vitaminas y antiparasitarios. La comercialización de los productos agrícolas y pecuarios se realiza en el mercado local (parroquia) en el $80 \%$ de los encuestados, el $10 \%$ a mercados cantonales y el $10 \%$ a centros de acopio (mercados nacionales).

\section{DISCUSIÓN}

Los resultados coinciden con la literatura citada sobre los aspectos físicos presentes en Chuquiribamba, donde resalta el relieve colinado y las elevadas pendientes que van del 20 al 100\% (Álvarez-Hernández, 2020).

Al analizar el suelo y relacionarlo con la producción agrícola cabe resaltar que existe una limitada productividad asociada al bajo contenido de macronutrientes (N, P, K) (Cárdenas-Navarro et al., 2004) presentes en el suelo y al inapropiado manejo de fertilizantes (orgánicos e inorgánico), por tanto, el uso del suelo agrícola se reduce día a día.

Nuestros resultados acerca del uso del suelo coinciden con la información presentada por el Gobierno Autónomo Descentralizado de Chuquiribamba el cual menciona que el mayor uso de suelo es pecuario y las actividades realizadas están en función de las características del suelo y el relieve (GADP Chuquiribamba y Constructora Consultora Inka Pirka, 2015).

Los productos obtenidos en las actividades económicas hacen posible que la zona de estudio sea considerada como fuente abastecedora de plantas aromáticas, hortalizas, legumbres y ganado a los mercados de Loja, Zamora y El Oro (Álvarez-Hernández, 2020). Sin embargo, la producción agropecuaria se ve afectada por escasa organización para la producción y/o comercialización y la parcelación de la tierra, este último causado por las herencias familiares que convierten a los terrenos en superficies cada vez más pequeñas para la siembra (Montaño, 2011) y al no incorporar los nutrientes al suelo hace que la producción vaya disminuyendo (Ciampitti García 2008).

En el desarrollo de las actividades productivas se cuenta con el sistema de Riego Aguarongo-Zañe, el mismo que se considera como la fuente de abastecimiento para el riego en el sector. A pesar de lo dicho, algunas formas de riego el riego por aspersión encontrado mayoritariamente en este trabajo o el reigo por gravedad que se practica en otras zonas de pendiente, conjuntamente con la inadecuada utilización del agua, genera problemas de lavado de suelos y déficit hídrico, la reducción del volumen de agua se agudiza notablemente en el período de verano (Flores Gallardo et al., 2014).

La población del área realiza actividades productivas que fueron transmitidas por sus ancestros; en regiones rurales, la pericia en la producción responde a los factores ambientales y a factores bióticos, y evidencia diferentes habilidades de respuesta a la situación económica (Víquez, 1994), razón por la cual, tanto la producción agrícola y ganadera se considera indispensable para la supervivencia de la comunidad. La 
tenencia de tierra en el área de influencia refleja la inequidad en el acceso a la tierra, lo cual se ha visto en otros estudios que puede ser un factor limitante a la hora de producir (Alvarado Vandecandelaere, 2011), ya que la producción termina siendo a pequeña escala.

Las actividades productivas son realizadas por el hombre, sin embargo, la presencia de la mujer es evidente en la toma de decisiones relacionadas con las mismas. Las mujeres suelen estar inmersas en los espacios comunitarios a través del desarrollo de actividades sociales, administrativas y de formación (Quijano Correa, 2003), lo cual supone un menor trabajo en campo pero una mayor implicación activa de la mujer en la comunidad.

El presente estudio presenta la caracterización agrosocioeconómica y biofísica del sistema de riego Aguarongo-Zañe, quedando pendiente futuros estudios de las diferentes formas de manejo del suelo con fines de producción agrícola y pecuaria que permita elevar la productividad del área de estudio.

\section{CONCLUSIONES}

Las características biofísicas del área de estudio hacen posible la realización de actividades pecuarias y en menor cantidad actividades agrícolas, estas últimas se ven afectadas por el deficiente manejo del entorno natural, especialmente de los suelos y por ende la producción decrece día a día.

\section{AGRADECIMIENTOS}

Al presidente de la Junta de Riego del Sistema de Riego Aguarongo - Zañe y a todos los 99 socios por su colaboración en el desarrollo de la investigación. A la Universidad Nacional de Loja por su interés en el desarrollo de la región Sur del Ecuador.

\section{FINANCIAMIENTO}

El presente estudio fue financiado por una entidad no oficial.

\section{REFERENCIAS}

Alvarado, M. y Vandecandelaere, A. (2011). Tenencia de la tierra e inequidad en el acceso a la tierra. Tierra urgente, 51-79.

Álvarez-Hernández, O. (2020). Agroclimate potential for the use of irrigation in Chuquiribamba parish, Loja, Ecuador. Revista Cubana de Meteorología, 26(3).

Barrios N., Castro A., Coria, L., González A., Martínez V. y Taddey D. (2007). La relación global-local. Sus implicancias prácticas para el diseño de estrategias de desarrollo. Red Académica Iberoamericana Local-Global-EUMEDNET. Recuperado de www.eumed.net/libros/2007a/259/index.htm.

Beretta, A., Silbermann, A., Paladino, L., Torres, D., Bassahun, D., Musselli, R., García-Lamohte, A. (2014). Análisis de textura del suelo con hidrómetro: modificaciones al método de Bouyoucus. Ciencia e investigación agraria, 41(2), 263-271.
Cárdenas-Navarro, R., Sánchez-Yáñez, J., FaríasRodríguez, R., Peña-Cabriales, J. (2004). Los aportes de nitrógeno en la agricultura. Revista Chapingo Serie Horticultura, 10(2), 173-178.

Ciampitti, I., García, F. (2008). Balance y eficiencia de uso de los nutrientes en sistemas agrícolas. Revista Horizonte A, 4(18), 22-28.

Domínguez Soto, J., Román Gutiérrez, A., Prieto García, F., Acevedo Sandoval, O. (2012). Sistema de Notación Munsell y CIELab como herramienta para evaluación de color en suelos. Revista mexicana de ciencias agrícolas, 3(1), 141-155.

Flores B., T. (2008) ¿Desarrollo sostenible o sustentable? Asociación Boliviana Pro defensa de la Naturaleza.

Flores-Gallardo, H., Sifuentes-Ibarra, E., Flores Magdaleno, H., Ojeda-Bustamante, W., Ramos-García, C. (2014) 91 Sistema De Riego Aguarongo - Zañe Chamba-Ontaneda C. R. Técnicas de conservación del agua en riego por gravedad a nivel parcelario. Revista mexicana de ciencias agrícolas, 5(2), 241-252.

Flores-Gallardo, H., Sifuentes-Ibarra, E., FloresMagdaleno, H., Ojeda-Bustamante, W., y Ramos-García, C. R. (2014). Técnicas de conservación del agua en riego por gravedad a nivel parcelario. Revista mexicana de ciencias agrícolas, 5(2), 241-252.

Flores Jibaja, A. (2019). Diagnostico agrosocioeconómico del caserío "Sabanas" del distrito de Huarango provincia de San Ignacio, departamento de Cajamarca-Perú 2016.

Gallopín, G. (2003). Sostenibilidad y desarrollo sostenible: un enfoque sistémico. En: Serie Medio Ambiente y Desarrollo, No. 64. División de desarrollo Sostenible y Asentamientos Humanos. Naciones Unidas-Cepal-ECLAC, Santiago de Chile.

Gobierno Autónomo Descentralizado Parroquial Chuquiribamba y Constructora Consultora Inka Pirka Cia. Ltda. 2015. "Plan de desarrollo y ordenamiento territorial de la parroquia Chuquiribamba, cantón y provincia de Loja. Chuquiribamba". Mayo 2015. Loja - Ecuador.http://chuquiribamba.gob.ec/index.php/parroquia/produ ctos.

Gobierno Provincial de Loja (2018). Proyecto: "Rehabilitación de las redes de distribución del sistema de riego Aguarongo-Zañe".

Gonzalez Insua, M., Battista, E., y Justianovich, S. H. (2020). Diseño para la Sustentabilidad y Sistema Producto Servicio Sustentable. Aportes para el desarrollo de competencias en el contexto Argentino. (Spanish). Cuadernos Del Centro de Estudios de Diseño y Comunicación, 24(115), 57-70. 
Guerrero Lázaro, J. M. (2019). Determinación de la capacidad de intercambio catiónico por el método del formaldehído.

Instituto Nacional de Estadística y Censos. (2010). Censo de población y vivienda. Censo de población y vivienda.

Instituto Nacional de Investigación Geológico Minero Metalúrgico. (2017). Hoja geológica 56.

Instituto Nacional de Meteorología E Hidrología (INAMHI). (2013). Anuarios Meteorológicos 1990-2013. (Ed.). Quito: In I. N. d. M. e. Hidrología, Ecuador .

Leff, E., Argueta, A., Boegue, E. y Porto, C (2002). "Más allá del desarrollo sostenible: la construcción de una racionalidad ambiental para la sustentabilidad: una visión desde América".En: La transición hacia el desarrollo sustentable. Perspectivas de América Latina y el Caribe. Universidad Autónoma latinoamericana: México: 477-576.

Martínez, M. (2019). Relación entre el nivel de crecimiento económico de Guatemala y el flujo de materia a partir de la información disponible para el período de 2010 a 2015. (Spanish). ECO Revista Académica, 21, 1.

Méndez Valdiviezo, E. (2016). Validación del método analítico walkley y black de materia orgánica en suelos arcillosos, francos y arenosos del Ecuador (Bachelor's thesis, Quito: UCE).

Montaño J. 2011. "Plan de desarrollo y ordenamiento territorial de la parroquia Chuquiribamba perteneciente al cantón Loja". Tesis de grado previa a la obtención del título de ingeniero en geología ambiental y ordenamiento territorial. Universidad Nacional de Loja, 237 p. http://192.188.49.17/jspui/handle/ 123456789/14796.

Mora, L. (2013, August). Dimensión ambiental, desarrollo sostenible y sostenibilidad ambiental del desarrollo. In Eleventh LACCEI Latin American and Caribbean Conference for Engineering and Technology (LACCEI'2013)“"Innovation in Engineering, Technology and Education for Competitiveness and Prosperity" August (pp. 14-16).

Quijano, M. y Correa, E. (2003). Mujeres rurales y nueva ruralidad en Colombia. Cuadernos de desarrollo rural, (51).

Ruales Torres, F. (2019). Determinación de la capacidad de intercambio catiónico del suelo y su correlación con el contenido de cationes intercambiables de las plantas del género Siparuna (Bachelor's thesis).

Ruiz Román, L. (2018). Validación de métodos de ensayo para determinación de $\mathrm{pH}$, conductividad, sólidos sedimentables, sólidos suspendidos y sólidos disueltos en aguas en el Laboratorio Ambiental Environovalab (Bachelor's thesis, Quito: UCE).
Vargas Rojas, R. (2009). Guía para la descripción de suelos (No. FAO 631.44 G943 2009). FAO, Roma (Italia).

Víquez, E. 1994. Caracterización del huerto mixto tropical “La Asunción”, Masatepe, Nicaragua. En: Agroforestería en las Américas. Vol. 1, N². p 5-9.

Zúñiga, M. Y. (2011). Sostenibilidad y Sustentabilidad como estrategias para crear valor en las viviendas actuales. Tesina de Especialidad en Ingeniería Civil (Construcción). Facultad de Ingeniería. Ciudad de México: UNAM. 